\title{
An Analytical and Experimental Investigation of Average Laser Power and Angular Scanning Speed Effects on Laser Tube Bending Process
}

\author{
Khalil Ibraheem Imhan ${ }^{1,3}$ B.T.H.T. Baharudin ${ }^{1}$, Azmi Zakaria ${ }^{2}$, Mohd Idris Shah b. Ismail ${ }^{1}$, Nasser Mahdi Hadi Alsabti ${ }^{3}$ \\ and Ahmad Kamal Ahmad ${ }^{4}$ \\ ${ }^{1}$ Department of Mechanical and Manufacturing Engineering, Faculty of Engineering, University Putra Malaysia, 43400 UPM Serdang- \\ Selangor Darul Ehsan-Malaysia \\ ${ }^{2}$ Faculty of Science, University Putra Malaysia, 43400 UPM Serdang-Selangor Darul Ehsan-Malaysia \\ ${ }^{3}$ Laser research center, Ministry of science and Technology, Baghdad, Iraq \\ ${ }^{4}$ Faculty of Science, Al-Nahrain University, Baghdad, Iraq
}

\begin{abstract}
Laser tube bending is a new technique of laser material forming to produce a complex and accurate shape due to its flexibility and high controllability. Moreover, the defects during conventional tube forming such as thinning, wrinkling, spring back and ovalization can be avoided in laser tube bending process, because there is no external force used. In this paper an analytical investigation has been conducted to analyses the effects of average laser power and laser scanning speed on laser tube bending process, the analytical results have been verified experimentally. The model used in this study is in the same trend of the experiment. The results show that the bending angle increased with the increasing of average laser power and decreased with the increasing of angular scanning speed.
\end{abstract}

\section{Introduction}

The laser forming has taken place during the last three decades due to its ability to produce accurate and complicated shapes with perfect controllability [1]. Nowadays, researches have been conducted and focused on laser tube bending due to its importance to different applications such as heat exchangers, automotive, aerospace, and medical field. Moreover, different aspects have been studied to understand the process behavior and the effect of the parameters especially laser and laser beam delivery. Hao, N. [2] proposed an equation for the bending angle taking into account plastic mechanics and validated by an experimental investigation. He reported that the bending angle is as a consequence of average laser power, laser angular scanning speed, tube measurements, and material properties. The average laser power and the scanning speed, in general, have the strong correlation with each other; hence, the produced bending angle mainly depends on these two factors and their effects on the rest aspects of the laser tube bending. Theoretically, the bending angle is directly proportional to the average laser power and inversely proportional to the angular scanning speed [3]. Moreover, in laser tube bending the ratio between the laser beam diameter and the tube thickness is considered high, and the angular scanning speed is low to have a close homogenous temperature distribution via the wall thickness direction for the duration of laser irradiation. Hsieh and Lin [4] solved the uncoupled thermal-mechanical problem in laser tube bending using a three-dimensional finite element method. Zhang et al. [5] investigated a numerical simulation for tube bending, where there are four lasers scanning schemes, involving point-source circumferential scanning, pulsed line-source axial procession, and linesource axial scanning without and with water cooling. It is clear that scanning schemes extremely impact laser tube bending. In another sentence, the laser beam diameter is also an influential factor in laser tube bending. Lawrence et al. [6] mentioned that the laser beam diameter is one of the parameters which have to be taken into account in the process design to avoid melting of the material surface. It also affects clearly on laser process mechanism. The general analytical expressions for tube bending are mostly circumferential scanning schemes assuming a two-dimensional temperature distribution and temperature independent material properties. In addition, there are other types of scanning such as axial and alternate circumferential [7]. Furthermore, Jie Zhang et al. [5] investigated a numerical simulation for tube bending, where there are four lasers scanning schemes, involving point-source circumferential scanning, pulsed line-source axial procession, and line-source axial scanning without and with water cooling. It is clear that scanning schemes extremely impact laser tube bending. Comparing the axial scanning scheme to the circumferential scanning scheme the angle produced is twice times; hence the number of scanning would be lesser. The mechanical method is used to bend tubes with a thin wall, but it is unable to bend large tube diameter more than 2 inch, due to difficulties of producing the templates and its high cost. Hence, the extrados portion suffers stretching and the intrados portion suffers from wrinkling rather than the distortion of the cross section [8]. Zhang et al. [9] suggested laser light to do such task and bend large tube diameter 6 inches with a thin wall, they refer to wall factor $>80$ 
which can define as the ratio between OD and wall thickness. Many techniques have been used to assist the laser forming process and overcome the challenges. Where plugs inserted inside the free end of the tube to avoid ovalization and active cooling was used to increase the process speed. Moreover, the tube surface is coated to enhance the absorptivity as well as the rectangular laser beam is utilized to produce a uniform thermal profile. Tube diameter over 12 inches has bent to 5 degrees after 200 scans with 20 minutes in $200 \mathrm{~mm}$ length. In this paper an analytical investigation has conducted to study the effects of average laser power and laser angular scanning speed on laser tube bending process, the analytical results have verified experimentally.

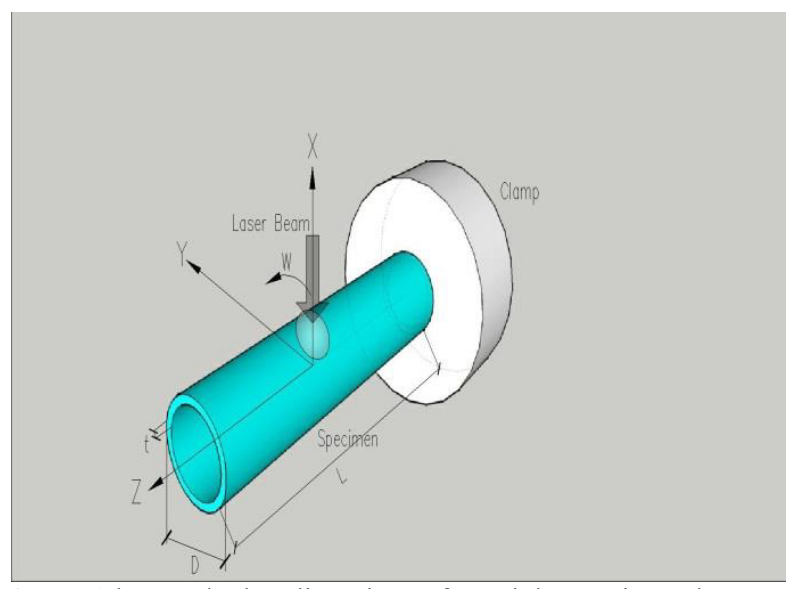

Figure 1 laser tube bending circumferential scanning scheme

\section{Analytical approaches}

Laser tube bending is considered a heat treatment process in a high level of temperature, but still the material surface temperature is less than the melting point. In order to understand the nonlinear phenomena accompany the process $\mathrm{Hao}$ and $\mathrm{Li}$ [10] established a new analytical model to determine the bending angle and studied the effects of significant parameters. Circular tube with circumferential scanning was used as shown in Fig. 1, the tube rotates on its axial axis around $180^{\circ}$, where the laser light is defocused on the tube surface with laser beam diameter much bigger than tube thickness. The heated area suffered wall thickening and compressive plastic deformation because of the thermal expansion limitation by the unheated material. When the laser source is turning off a rapid cooling, occur, with material shrinkable on the heated surface. As a result, the shortening of irradiated material in the axial of the tube direction forced it to bend towards of laser source. During bending process, heat is generated because of strain energy, but it is very small compared to input laser beam energy so it can be neglected. The importance of the analytical solution and mathematical representation is obvious in understanding the laser tube bending process behavior and diagnosis the parameters effects. Hao and Li [10], [11] Explicates the laser tube bending and create an analytical model that can involve the effects of laser, material, and geometry parameters to find the bending angle $\theta$, as well as they, used numerical analysis to simulate the process. The laser is heating the material surface and induced plastic compressive strain, from Fig. 2 there are two Force's F exerts inversely to each other between the heated and unheated material where the laser scanning is only on the upper portion of the tube circumference and in the axial direction.
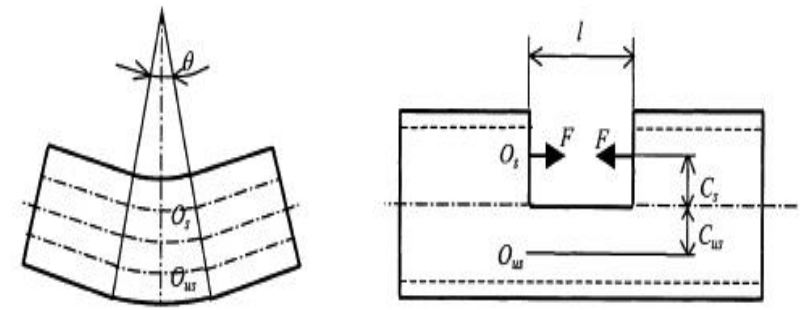

Figure 2. Simplified model for laser tube bending

Strain on scanning area is:

$$
\varepsilon_{s}=-\Delta T \alpha / 2+F / S E
$$

where $S$ is the area of the section of the half tube. $E$ is the Yong's modulus of the material.

The strain of unscanned portion is

$$
\varepsilon_{u s}=-F / S E
$$

The angle bend can be found from the below:

$$
\theta=(\pi l / 4 D)\left[\Delta T \alpha-2(\sqrt{ } 2-1) \sigma_{y} / E\right]
$$

where $l$ is the length of heated region and can be considered the laser beam diameter, $D$ is a tube diameter, and $\sigma_{y}$ is a yield stress of the material.

The heat energy generated by laser for bending is:

$$
E=P \eta v t^{\prime}
$$

where $P$ is a laser power, $\eta$ is the absorption coefficient $v$ is the ratio of heating power to input power, and ' $'$ ' is a time period of laser scan.

From all the above the laser tube bend angle can be written as:

$$
\theta=(\pi l / 2 D)[(P \eta v \alpha / \omega D l t \rho c)-(\sqrt{ } 2-1) \sigma y / E]
$$

where $\omega$ is an angular scanning speed, $\rho$ is a density of the material, $t$ tube thickness, and $c$ is a specific heat of the material.

\section{Laser parameters}

The laser parameters are the most important among other parameters in laser tube bending process due to its being flexible and can be controlled in many aspects of its properties. All the literature mentioned that the effect of laser power is linearly proportional to the value of angle produced, whilst the scanning speed has inverse proportionality. In order to be more specified, one has to study the laser beam and scanning characteristics[12]. The general analytical expressions for tube bending are mostly circumferential scanning schemes assuming a twodimensional temperature distribution and temperature independent material properties. 


\section{Experiment setup}

A high power pulsed Nd-Yag laser JK300HPS of Maximum Average Power Laser 300 (W) emitting at $1064 \mathrm{~nm}$ and fiber coupled has been used to irradiate stainless steel 304 (SS304) tubes of diameter $12.7 \mathrm{~mm}$, $0.6 \mathrm{~mm}$ thickness and $60 \mathrm{~mm}$ in length. Moreover, computerized RSA Series Motorized Rotation Stage with its controller (from Zolix Company) is used for holds and rotates the tube as illustrated in Fig. 3. Hence, the tube is irradiated with different average laser power range from 0 to $200 \mathrm{w}$ of laser beam diameter $7.5 \mathrm{~mm}$; the angular scanning speed is 20 to $40 \mathrm{deg} / \mathrm{sec}$ and rotated angle $180^{\circ}$. The deviation of the tube around the $\mathrm{x}$-axis is measured by dial gauge to determine the bending angle $\theta$. Furthermore, the following specification of the SS404 has used, the yield stress of $205 \mathrm{MPa}$, density $7930 \mathrm{~kg} / \mathrm{m} 3$, Young's modulus of $193 \mathrm{GPa}$, the coefficient of thermal expansion of $17.5 \times 10-6 \mathrm{k}^{-1}$, the specific heat of 500 $\mathrm{J} / \mathrm{Kg} . \mathrm{K}$, the absorption coefficient $\eta$ supposed to be 0.6 [13]. On the other hand, the ratio of heating power to input power $v$ depends on many conditions such as the heat losses due to convection and radiation which is a function of the material, scanning speed and geometry specification. In this study, $v$ is set 0.8 and extreme care is paid to avoid surface melting of the SS 304 during the bending processes.

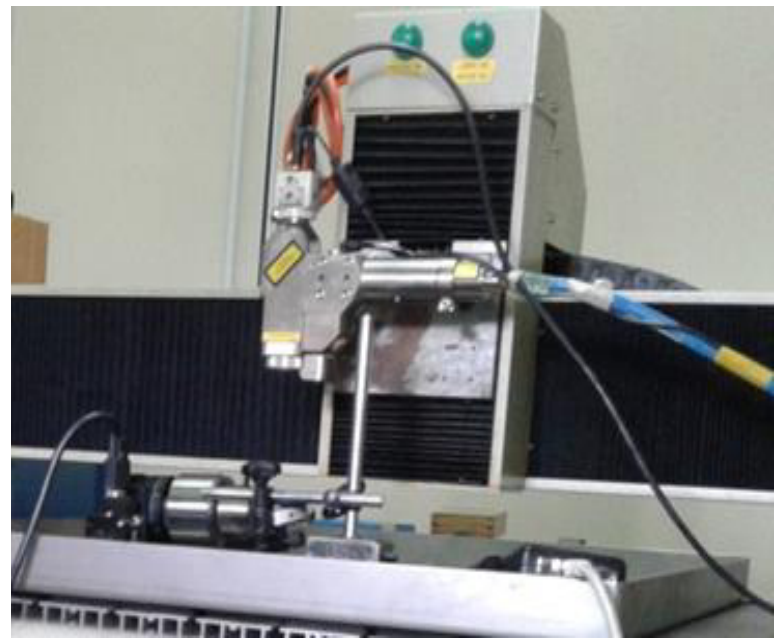

Figure 3. Experiment setup

\section{Results and discussions}

From the relationship between the laser power and the bending angle in both cases, the bending angle increases with the increasing of average laser power as illustrated in Fig. 4. The bending angle in analytical results is higher than the experimental results, especially at the low average laser power amount. This is due to the nonlinear absorption of the material to the laser power, but it is increased with the increasing of the laser power and the gap become very small when the laser power reaches 200 W. With temperature increasing, absorption coefficient increases also other temperature dependency properties such as thermal expansion coefficient and specific heat, hence more of laser energy is penetrated inside the material [14].

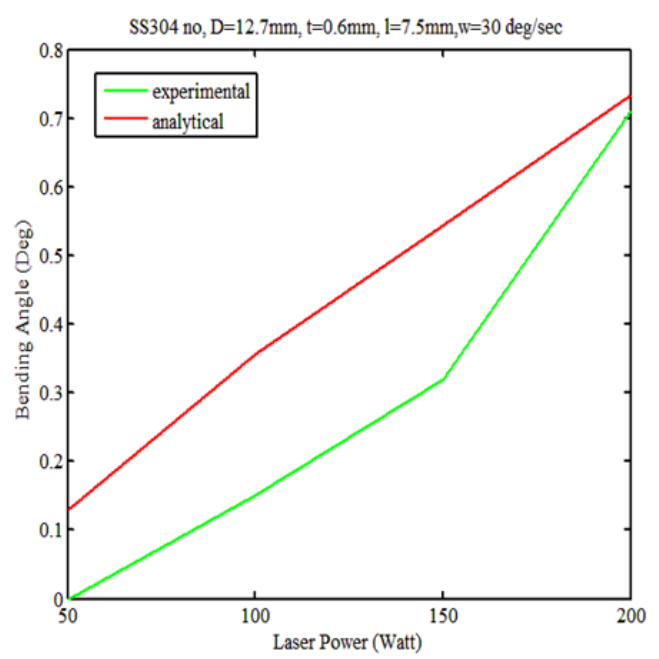

Figure 4 Relationship between average laser power and bending angle

The maximum bending angle is become close to 0.7 degree once the analytical and the experimental using average laser power $200 \mathrm{~W}$. the analytical and the experimental results are in the same trend in this level of average laser power and when the angular scanning speed hold steady on $30 \mathrm{deg} / \mathrm{sec}$.

On the other hand, Fig.5 shows the relationship between the angular scanning speed and the bending angle of analytical and experimental studies which in the same trend but a different slope. As the angular scanning speed decreases as the bending angle increased [3], analytically the bending angle can reach to $3^{\circ}$ if the scanning speed is reduced to $5 \mathrm{deg} / \mathrm{sec}$. However, experimentally that cannot be achieved because the material temperature increased and reach to the melting point and the tube collapses. Furthermore, when the angular scanning speed decreased the time for energy absorption is increased by the material which enhances the process. In Fig. 5 the average laser power $200 \mathrm{~W}$ was used, when the scanning speed has reduced to $20 \mathrm{deg} / \mathrm{sec}$ the experiment bending angle is about $30 \%$ bigger than the analytical bending angle. Hence, the experiment results are higher than analytical due to the increase of absorption coefficient.

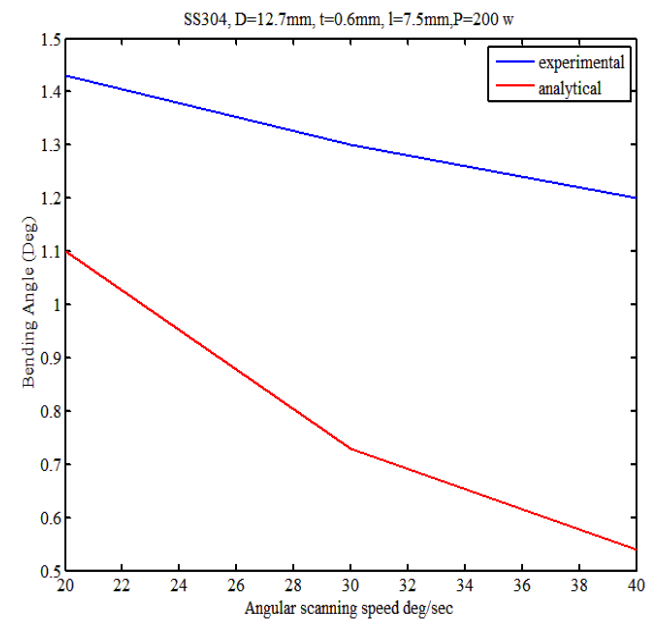

Figure 5. relationship between angular scanning speed and bending angle 


\section{Conclusions}

Analytical and experimental investigations were conducted to study the effect of the average laser power and angular scanning speed on the laser tube bending process. Moreover, the analytical model depends mainly on the average laser power and angular scanning speed because those parameters can be changed during the process in spite the effects of other parameters are present. The analytical results are verified by sets of experiments and hence the conclusions can be listed as below:

- The angle bending $\theta$ is proportional directly to the average laser power.

- The angular bending $\theta$ is proportional reversely to the angular scanning speed.

- The analytical results are in acceptable agreement with the experiments results.

- The average laser power has to match with the angular scanning speed to avoid the scraping effect on the specimen surface.

\section{References}

1. Kannatey-Asibu Jr, E., Principles of laser materials processing. Vol. 4: John Wiley \& Sons. (2009).

2. Hao, N. On the process parameter of laser tube bending. in 2010 International Conference on.Mechanic Automation and Control Engineering (MACE), (2010)

3. Safari, M. and M. Farzin, A study on laser bending of tailor machined blanks with various irradiating schemes. Journal of Materials Processing Technology,. 214(1): p. 112-122, (2014).
4. Hsieh, H.-S. and J. Lin, Study of the buckling mechanism in laser tube forming. Optics \& Laser Technology. 37(5): p. 402-409, (2005).

5. Zhang, J., et al., Effects of scanning schemes on laser tube bending. Journal of manufacturing science and engineering. 128(1): p. 20-33, (2006).

6. Lawrence, J.R., Advances in laser materials processing: Technology, research and application.: Elsevier, (2010).

7. Safdar, S., et al., Finite element simulation of laser tube bending: Effect of scanning schemes on bending angle, distortions and stress distribution. Optics \& Laser Technology. 39(6): p. 1101-1110, (2007).

8. He, Y., et al., Advances and trends on tube bending forming technologies. Chinese Journal of Aeronautics. 25(1): p. 1-12, (2012).

9. Zhang, W., et al. Large diameter and thin wall laser tube bending. in 24th International Congress on Applications of Lasers and Electro-Optics, ICALEO. (2005).

10. Hao, N. and L. Li, An analytical model for laser tube bending. Applied surface science. 208: p. 432-436, (2003).

11. Hao, N. and L. Li, Finite element analysis of laser tube bending process. Applied surface science. 208: p. 437-441, (2003).

12. Jamil, M.C., M. Sheikh, and L. Li, A study of the effect of laser beam geometries on laser bending of sheet metal by buckling mechanism. Optics \& Laser Technology. 43(1): p. 183-193, (2011).

13. Davis, J.R., Stainless steels. 1994: ASM international.

14. Li, W. and Y.L. Yao, Laser bending of tubes: mechanism, analysis, and prediction. Journal of manufacturing science and engineering. 123(4): p. 674-681, (2001). 